\title{
Measurement and Recording of Reflective Properties of Materials
}

\begin{abstract}
The aim of this paper is to analyse possibilities of measurement of reflective properties of materials used in the interior and to propose methods of recording of measured reflective properties of materials for calculation of multiple reflections in light scenes. One of the basic assumptions for definition of the appropriate methods of recording of measured reflective properties of materials is the analysis of input data and methods used for the calculation of multiple light reflections in computer graphics and capabilities of these methods in the lighting calculations.
\end{abstract}

Keywords: lighting, reflective properties of materials, multiple reflections.

\section{Introduction}

Idealized reflective surfaces (diffuse and specular) are currently used to describe the reflective properties of the materials. Lighting design calculation programs are replacing the lighting model with ideally diffuse surfaces, because they provide simplification of calculations by using radiosity calculation method. This simplification leads to suppression of specular reflection component and causes inaccurate calculation results, because real materials are characterised by reflective properties different from idealized cases.

Calculation methods used in computer graphics (such as ray tracing) can achieve more accurate results of multiple light reflections calculation, because these methods calculate with real reflective properties of surfaces in the scene. Therefore, it is necessary to design an appropriate method for measuring and especially recording of reflective properties of real materials into data files so that they can be used for lighting calculations using methods of computer graphics or architectural visualization and to compare these results of the calculations with the results of commonly used lighting calculation programs.

\section{Measurement of reflective properties of materials}

When studying the reflective properties of real materials it is necessary to perform the luminance analysis of the real material surface (secondary light source), that requires extensive luminance measuring of sample surfaces depending on the angle of light incidence from point light source. Measured values of the luminance of the sample $L_{r}(\alpha, \gamma)$, depending on the angle of incidence $(\beta)$ of light, is necessary for the description of the luminance indicatrix of the surface and for construction of bidirectional reflectance distribution function, which is most frequently used to describe the reflective properties of the materials in the architectural visualization programs. Luminance analysis of the secondary light source (material sample) is based on the use of a photometric bench which allows to illuminate the sample of material with point light source from various angles of incidence $(\beta)$ and simultaneously measuring the luminance $L_{r}(\alpha, \gamma)$ of the surface of the sample at various angles of light reflection $(y)$, and with various rotation of the sample relative to the projection of the line light source material sample to the horizontal plane (angle $\alpha$ ). The arrangement of a workplace with fixed luminance meter in combination with adjustable and rotatable material sample and light source is shown in Fig. 1.

The number of measurement positions of described device is determined by measuring step $\Delta \alpha=\Delta \beta=\Delta y=10^{\circ}$ for all three observed angles $\alpha, \beta, \gamma$ and by the measuring range of angles $\alpha \in\left\langle 0^{\circ} ; 360^{\circ}\right\rangle ; \beta \in\left\langle 0^{\circ}, 70^{\circ}\right\rangle ; \gamma \in\left\langle 0^{\circ}, 80^{\circ}\right\rangle$ These conditions leads to 2664 various combinations of angles for each measured sample. Angles of incidence $\beta$ and angle of reflection $\gamma$ could not be set to $90^{\circ}$ (resp. $80^{\circ}$ ), because at these angles the fixing aperture frame, respectively arm construction with a light source, is causing obscuration of the surface sample.

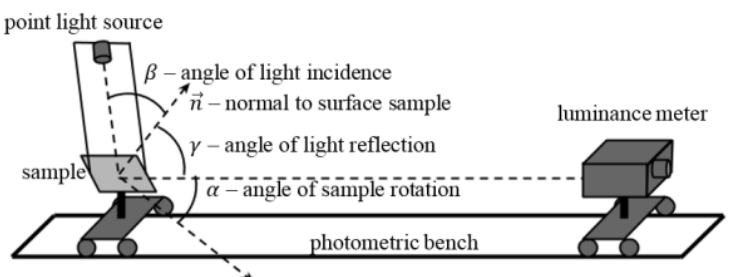

projection of the line light source - material sample to the horizontal plane

Fig.1. Photometric bench with the device for luminance analysis of reflected light

For the measurement was chosen a sample of material widely used in the interiors - lacquered wood surface - which represents various types of furniture and which also represents the best distinction from the idealized models of light reflection. Such materials should ideally exhibit neither ideal diffuse nor ideal specular reflection, but absolutely general reflective properties.

The following figure (Fig. 2) shows luminance diagram of the measured material sample in polar coordinates corresponding to each angle of light incidence $\beta$ and to the sample rotation angles $\alpha=0^{\circ}$. Dashed line shows the direction of light incidence for the corresponding colour of luminance diagram.

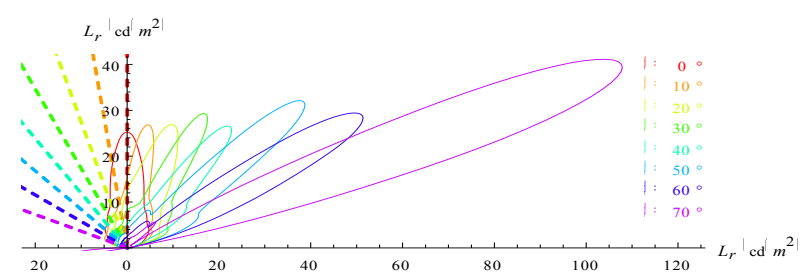

Fig.2. Luminance polar diagram of the measured material sample in polar coordinates corresponding to angles of light incidence $\beta$ and to the sample rotation angle $\alpha=0^{\circ}$

\section{Analysis of reflective properties of the materials}

When dealing the process of multiple light reflections, it is important to determine the proportion of the total reflected luminous flux $\Phi_{\rho}$ and luminous flux $\phi_{i}$ incident to the reference point $P$ of the material sample. This can be realized using equation (1) for integral reflectance $\rho$ calculation based on determined values of sample illuminance $E_{P}$ and sample 
luminous exitance $M_{P}$ at point $P$ in combination with the equation (2) for luminous exitance $M P$ used for calculation of luminous exitance of a sample on the basis of knowledge luminance indicatrix of the sample $L_{r}(\alpha, \gamma)$ [1].

$$
\begin{gathered}
\rho=\frac{\Phi_{\rho}}{\Phi_{i}}=\frac{M_{P}}{E_{P}} \\
M_{P}=\int_{0}^{2 \pi} L_{r}(\alpha, \gamma) \cdot \cos \gamma \cdot d \Omega_{r}=\frac{\Phi_{\rho}}{A}
\end{gathered}
$$

When the material sample is illuminated by a point light source, it is necessary to determine the integral reflectance for each angle of incidence of light. From these individual values of integral reflectance can be subsequently determined their mean value characterizing the reflective properties of the material independently on the direction of light incidence.

Illuminance at material sample $E_{P O}$ with an angle of light incidence $\beta=0^{\circ}$ was measured using luxmeter Minolta T-10 and its corrected value (according to the verification certificate of luxmeter) is $E_{P O}=82.6 \mathrm{Ix}$. Measured values of illuminance of the sample by point light source and the luminance indicatrix $L_{r}(\alpha, \gamma)$ are needed for the calculation of bidirectional reflectance distribution function $f_{B R D F}(\alpha, \beta, \gamma)$, see equation (3).

$$
f_{B R D F}=\frac{d L_{r}(\alpha, \gamma)}{d E_{P}(\beta)}
$$

Integral luminous exitance $M_{P}(\beta)$ of the sample has been determined for each angle of light incidence $\beta$ using method derived from the method of zonal flux calculation, which is used to determine the luminous flux of the light source from spatial luminance diagram. Applying equation (1) it was determined integral reflectance $\rho$ for each measured angle of incidence $\beta$. Mean value of reflectance of the material sample calculated using described method is $\rho=0.4$.

Luminous intensity $l_{\alpha \gamma}$ of the material sample at various viewing angles $(\alpha, \gamma)$ can be calculated from the measured luminance values $L_{r}(\alpha, \gamma)$ using equation (4).

$$
I_{\alpha \gamma}=L_{r}(\alpha, \gamma) \cdot \cos \gamma
$$

Calculated values of luminous intensity $I_{\alpha \gamma}$ are shown in the following figure (Fig. 3) in polar coordinates corresponding to the various angles of light incidence $\beta$ for angles of rotation $\alpha=0^{\circ}$. Dashed line shows the direction of light incidence for the corresponding colour of luminous intensity diagram.

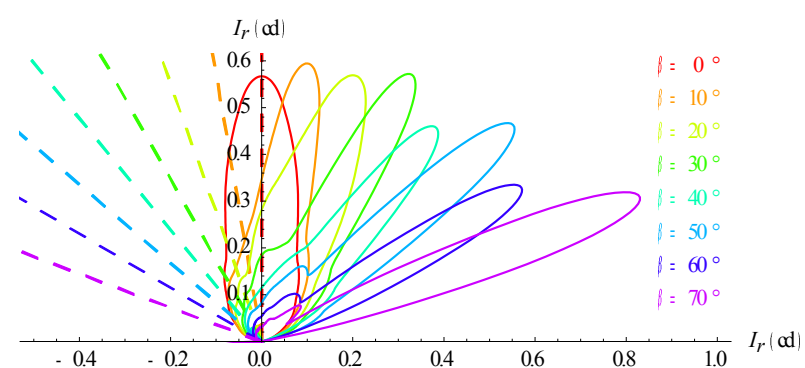

Fig. 3. Luminous intensity polar diagram of the measured materia sample in polar coordinates corresponding to angles of light incidence $\beta$ and to the sample rotation angle $\alpha=0^{\circ}$
Bidirectional reflection distribution function $f_{B R D F}(\alpha, \beta, \gamma)$ of the material sample under different viewing angles $(\alpha, \gamma)$ can be calculated from measured luminance values $L_{r}(\alpha, \gamma)$ and measured illuminance values of the material surface using equation (3).

Calculated values of bidirectional reflectance distribution function $f_{B R D F}(\alpha, \beta, \gamma)$ describing the measured material sample are shown in the following figure (Fig. 4) for various angles of light incidence $\beta$ and sample rotation angles $\alpha=0^{\circ}$. Dashed line shows the direction of light incidence for the corresponding colour of bidirectional reflectance distribution function diagram.

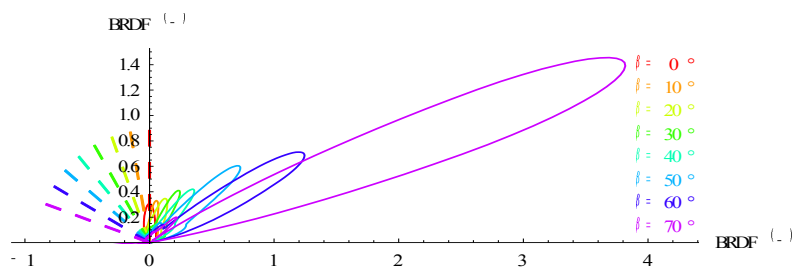

Fig. 4. Bidirectional reflectance distribution function polar diagram of the measured material sample in polar coordinates corresponding to angles of light incidence $\beta$ and to the sample rotation angle $\alpha=0^{\circ}$

\section{Recording of the reflective properties of materials}

The most appropriate method of recording of the measured reflective properties of materials is based on proposed future practical use of these data in computer programs for lighting design and technical programs for realistic scenes rendering (visualization).

The input data in commonly used programs for designing lighting systems are Eulumdat files that contain a description of photometric parameters of the luminaires (created on the basis of the calculated values of luminous intensity of the sample). The methodology for creating Eulumdat files is based on known procedures [2] and is described in detail in [3]. Eulumdat files created on the basis of the calculated values of luminous intensity of the material sample were used for the method of calculation of single light reflection on the surface called equivalent luminaires in [3].

Programs for architectural visualization work with bidirectional reflection distribution functions. To record the values of bidirectional reflectance distribution function it was proposed methodology derived from the procedure used to describe the light transmission and reflection of a plane window systems with blinds, for which there is a system of internal and external distribution hemisphere called Klems patches [4]

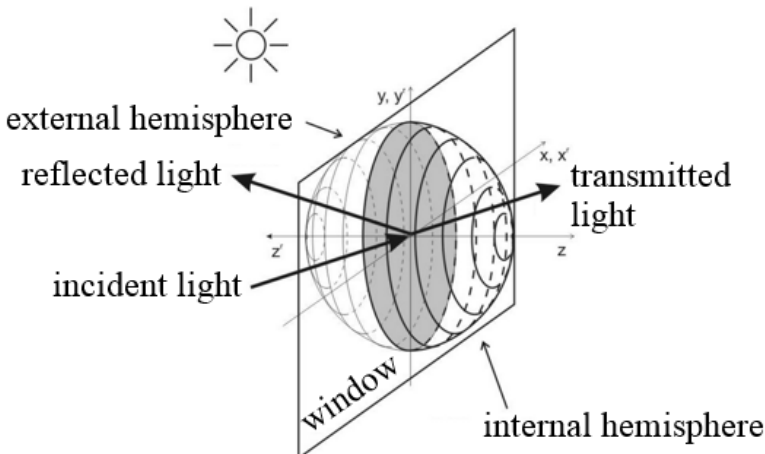

Fig. 5. Internal and external window hemisphere [5]

Klems patches define a division of each of described hemispheres (internal and external) to 145 partial spatial angles (patches) for angles $\alpha$ and $y$ in the external 
hemisphere (or $\alpha_{t}$ and $y_{t}$ in the internal hemisphere) so that if the illumination from all directions of the hemisphere to the control point $P$ is uniform $\left(L_{r}=\right.$ const.), the illuminance at a point $P$ is approximately the same from the point light source placed in any direction [6].

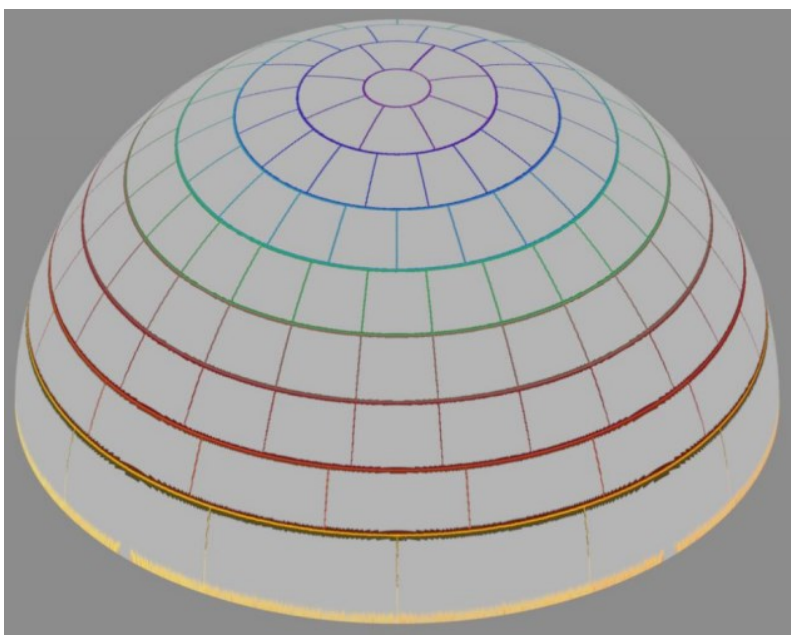

Fig.6. Hemisphere division to 145 Klems patches [7]

Each of the Klems patches characterizes partial spatial angle (the direction of its axis), from which the light (radiation) comes onto the surface of the reference sample (source patch), or into which is reflected (reflection patch) or transmitted (transmission patch). Therefore luminance analysis starts with positioning the point light source to the axis of each of 145 source patch, and then the luminance at the centre of the measured surface sample is measured in the axis of each of all 145 segments (reflection or transmission patch).

For each pair of source and reflection patches (or source and transmission), the value of bidirectional reflectance distribution function $f_{B R D F}(\alpha, \beta, \gamma)$ is calculated according to equation (3). For each of the 145 source patches is thus determined 145 values of bidirectional distribution function [8]. The result of such analysis is a matrix of function values of the size $145 \times 145$ (i.e. 21,025 functional values) where the columns correspond to the order of the source patches and the lines correspond to reflection or transmission patches. Order of segments in the matrix is determined by their serial numbers listed in [3] and are graphically indicated in Fig. 7.

The matrix of functional values of bidirectional distribution functions corresponding to the individual patches in Klems system can be recorded into data files using XML standard (Extensible Markup Language), which can be used for material description in programs for architectural visualization [6].

Values of bidirectional distribution functions recorded using the methodology described in XML format can be graphically displayed using a browser BSDFViewer for each source patch in the form of a diagram showing the top view (in the direction of normal to the reference surface) of the Klems patches. BSDFViewer Browser allows the user to select the source patch from the hemisphere (Incident Hemisphere) for which is graphically displayed the distribution of relative luminous flux reflected from the material sample after illumination of the sample by point light source placed in the direction defined by the selected source patch. Sample values of bidirectional reflectance distribution function of the material sample recorded in the system of Klems patches are shown in Fig. 7 and Fig. 8.

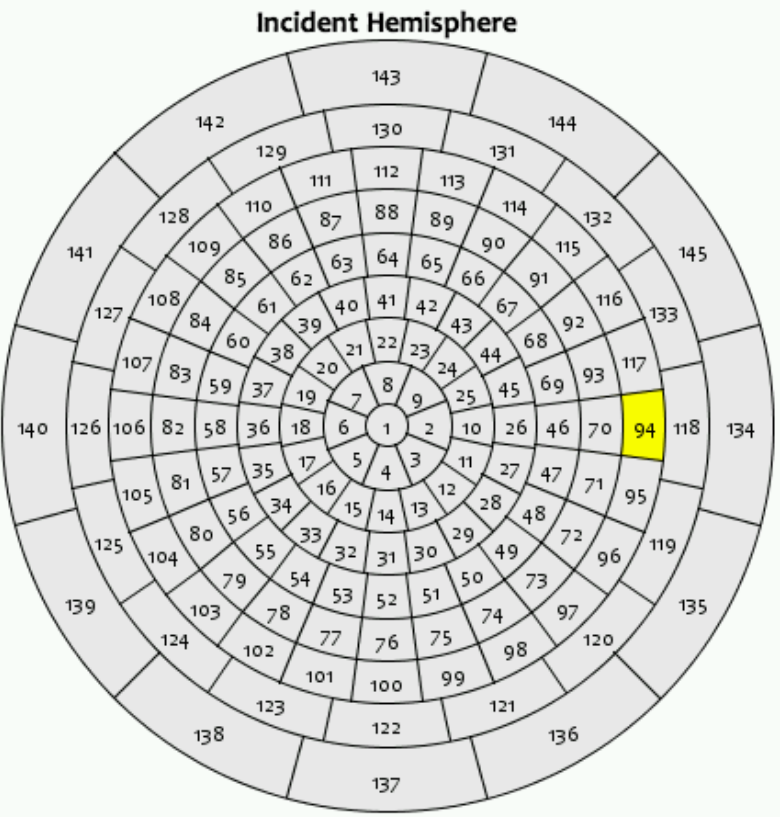

Fig. 7. The serial numbers of patches when viewed from the top of Klems system; patch no. 94 selected to display data in BSDFViewer

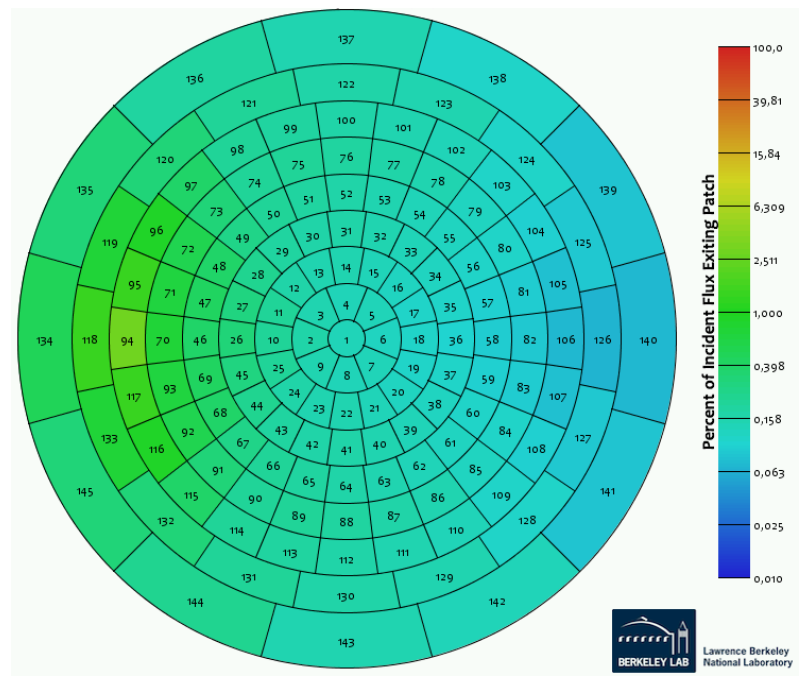

Fig. 8. Relative values of the spatial distribution of luminous flux reflected from described material sample illuminated from a point source located in the selected segment no. 94

\section{Conclusion}

The measurement device for luminance analysis of the material surface sample was constructed within this research. The spatial luminance distribution of the material sample was measured at the photometric bench using described measurement device. To describe real reflective properties of materials it is necessary to perform a very accurate measurement of the spatial luminance distribution of the material surface.

Two methods of luminance values recording for further use were designed based on the research of advanced methods for multiple light reflections calculation. Measured luminance values were using described methods subsequently converted to luminance indicatrix and to bidirectional reflectance distribution function (recorded in XML format), which is used as the input data for advanced methods of light calculations. A necessary condition for achieving accurate results of interior lighting calculations is to use the real models of reflective properties of materials 
measured with such device described in this research and to work with advanced light calculation methods that allow to calculate with real models of reflective properties of materials recorded with proposed methods.

The research presented in this paper was supported by the grant of Czech Technical University in Prague no. SGS13/197/OHK3/3T/13.

\section{REFERENCES}

[1] J. Habel a kol., Světlo a osvětlování, Praha: FCC Public, 2013. ISBN: 978-80-86534-21-3.

[2] „EULUMDAT File Format Specification““ Heart Consultants Limited, 1999-2012. [Online]. Available at: http://www.helios32.com/Eulumdat.htm. [Přístup získán 125 2014].

[3] M. Bálský, Analýza procesu mnohonásobných odrazů, Praha: ČVUT v Praze, FEL, disertační práce, 2014

[4] J. Klems, ,A New Method for Predicting the Solar Heat Gain of Complex Fenestration Systems," ASHRAE Winter Meeting, New Orleans, 1994.
[5] Carli, Inc., „Calculation of optical properties for a venetian blind type of shading device, “ Millers Falls, 2006.

[6] D. Geisler-Moroder, „BSDF Crash Course and The Radiance 3Phase-Method," 11th International Radiance Workshop, Copenhagen, 2012.

[7] M. Saxena, G. Ward, T. Perry, L. Heschong a R. Higa, „Predicting Annual Daylight with Variable Fenestration Optics using BSDFs,“ Fourth National Conference of IBPSA-USA, New York City, 2010.

[8] J. H. Klems a J. L. Warner, „Measurement of Bidirectional Optical Properties of Complex Shading Device,“ Lawrence Berkley Laboratory, University of California, Berkley, 1995.

Authors: ing. Marek Bálský, prof. ing. Jiři Habel, DrSc., Czech Technical University in Prague, Faculty of Electrical Engineering, Technická 2, Praha 6, Czech Republic, e-mail: marek.balsky@fel.cvut.cz; habel@fel.cvut.cz 